\title{
The impact of pregnancy in an autoimmune disease
}

\begin{abstract}
Psoriatic arthritis is a chronic inflammatory disease associated with psoriasis. Arthritis has a significant impact on quality of life in patients with psoriasis. Studies on pregnancy and birth outcomes in women with psoriasis are insufficient with varying results. Pregnancy is associated with hormonal changes in estrogens and progesterone modifying the immune response. Various hormonal states are known to be associated with improvement and worsening of psoriasis and PsA.

A 34-year-old woman with psoriasis since 2006 presents persistent inflammatory lower back pain, stiffness, nail pitting, small psoriatic lesions on her feet, left sacroiliac pain and limitation of motion of the lumbar spine. She was diagnosed with psoriatic arthritis and started NSAID with good clinical response. She got pregnant and presented worsening of lower back pain and spreading of psoriatic lesions throughout the lower limbs. She started topical emollients and low-dose systemic steroid with good response during 8 weeks. During the remaining months of pregnancy, psoriatic lesions slowly disappeared and lower back pain improved. On postpartum period she presented worsening of lower back pain, a BASFI score of 8.3 and a BASDAI score of 6.6. At this moment, she is awaiting hospital approval for biological therapy.
\end{abstract}

Controlling disease activity with immunosuppressive agents is often necessary to achieve a remission or a low or minimal disease activity state.

Keywords: Pregnancy, psoriatic arthritis, psoriasis, sacroiliitis, lower back pain, autoimmunity, immunosuppression
Volume 5 Issue 5 - 2019

\author{
Mariana Formigo, Cristina Silva, Olinda \\ Miranda, Ana Gonçalves, Sara Freitas, Glória \\ Alves, Jorge Cotter \\ Internal Medicine Department, Hospital Senhora da Oliveira, \\ Portugal
}

\begin{abstract}
Correspondence: Mariana Formigo, Internal Medicine Department; Hospital Senhora da Oliveira; Rua dos Cutileiros, I I4, 4835-044 Creixomil, Guimarães, Portugal, Tel 0035 I 253540330, Email mariana.formigo@gmail.com
\end{abstract}

Received: September 09, 2019 | Published: September 23, 2019
Abbreviations: AS, ankylosing spondylitis; BASDAI, bath ankylosing spondylitis functional index; BASFI, bath ankylosing spondylitis functional index; bDMARD, biologic disease-modifying antirheumatic drug; CRP, creactive protein; ERS, erythrocyte sedimentation rate; HCQ, hydroxychloroquine; MRI, magnetic resonance imaging; PsA, psoriatic arthritis; RF, rheumatoid factor.

\section{Introduction}

Psoriatic arthritis is a chronic inflammatory musculoskeletal disease associated with psoriasis, usually exhibiting peripheral arthritis, dactylitis, enthesitis, and spondylitis. ${ }^{1,2}$ Nail lesions (pitting and onycholysis) are present in $80-90 \%$ of patients with PsA. The incidence of PsA is 6 per 100,000 per year and the prevalence is 1-2 per 1,000 in the general population. ${ }^{1}$ The annual incidence of PsA among patients with psoriasis is $2.7 \%$, and the prevalence of PsA in patients with psoriasis varies between $6 \%$ and $41 \%{ }^{1}$ In the majority of patients, psoriasis frequently precedes the development of arthritis. ${ }^{1,2}$ PsA diagnosis is established based on clinical history, physical examination and joints imaging, there are no specific tests or biomarkers. ${ }^{1,2}$ Radiographs usually reveal evidence of asymmetric sacroiliitis, spinal or peripheral joints diseases, as well as erosions, new bone formation and ankylosis. ${ }^{2}$ The Classification of Psoriatic Arthritis (CASPAR) criteria $^{3}$ may help establishing the correct diagnosis. ${ }^{1}$ Arthritis greatly affects the quality of life of patients with psoriasis, therefore treatment should be initiated as soon as possible in order to delay or stop joint damage. ${ }^{4}$

Studies on pregnancy, post-partum and birth outcomes in women with psoriasis or PsA are insufficient with varying results. Pregnancy is associated with hormonal changes in estrogens and progesterone modifying the immune response. During pregnancy and post-partum period, women with psoriasis and PsA frequently experience a change in their symptoms and clinical manifestations. ${ }^{4,5}$ Adverse neonatal outcomes may be associated with high disease activity during pregnancy. Therefore, achieving remission or minimal disease activity state with immunosuppressive agents is usually needed during pregnancy to prevent adverse outcomes. ${ }^{6}$

\section{Case report}

A 34-year-old woman with psoriasis since 2006 and positive family history (father diagnosed with AS and a brother diagnosed with psoriasis) had persistent inflammatory lower back pain (10/10grade) and stiffness (30minutes in the morning) for the last 16 years. On consultation, she exhibited nail pitting, small psoriatic lesions on her feet $(2-3 \mathrm{~cm}$ lesion in her plantar region), left sacroiliac pain, positive Patrick's test and limitation of motion of the lumbar spine. She presented radiological sacroiliitis on MRI and high levels of inflammatory markers as CRP $(6.4 \mathrm{mg} / \mathrm{dL}$, normal $<2.9 \mathrm{mg} / \mathrm{dL})$ and ERS $(42 \mathrm{~mm}$, normal $<20 \mathrm{~mm})$. According to Caspar Criteria ${ }^{3}$, she exhibited current psoriasis (2points), RF negativity (1 point), typical psoriatic nail dystrophy (1 point), reaching 4 points suggesting psoriatic arthritis (spondylitis predominant). She was medicated with NSAID. On second consultation, one month later, lower back pain responded to NSAID and she had no pain (0/10), no stiffness and no progression in dermatological lesions. She said she wanted to get pregnant that year and started HCQ, maintaining good clinical response. On the next consultation she was 12 weeks pregnant, exhibiting worsening of lower back pain (10/10) and spreading of psoriatic lesions throughout the lower limbs. She started topical emollients and low-dose systemic steroid (prednisolone $5 \mathrm{mg} /$ day) with good response during 8 weeks. 
During the remaining months of pregnancy, psoriatic lesions slowly disappeared and lower back pain improved (2/10), under treatment with HCQ. On postpartum period, she presented severe lower back pain (10/10), a BASFI score of 8.3 (Functional Impairment) and a BASDAI score of 6.6 (Active Disease). At this moment, she is awaiting hospital approval for biological therapy.

\section{Discussion/conclusion}

Recent studies suggests that disease flares are relatively common during pregnancy in autoimmune inflammatory conditions. ${ }^{6}$ Most pregnant women with psoriasis experience a change in their cutaneous disease, approximately $55 \%$ of women improving, $21 \%$ remaining stable and $23 \%$ worsening their clinical manifestations. ${ }^{5,7}$

Hormonal changes in pregnancy are important in modifying the immune response. When estrogen and progesterone levels drop down in periods as post-partum, prior to menses or menopause, psoriatic worsening has been reported. Estrogens are both immunosuppressive and immunostimulatory, stimulating B-cell-mediated immunity and suppressing T-cell-mediated immunity. ${ }^{8}$ Instead, progesterone is primarily immunosuppressive, down-regulating T-cell proliferative response and being essential in uterine immunosuppression, therefore high levels of progesterone have been associated with improvement of psoriasis. ${ }^{8}$ Another study involving women with PsA suggests that highest disease activity state was at 6 months postpartum. ${ }^{9}$

The heterogeneity of PsA requires a multidisciplinary and multispecialty approach to assess its multiple domains and identify individual treatment for every patient. ${ }^{10}$ Due to long-term outcomes of joint involvement in PsA inflammation, remission or, if remission cannot be achieved, a low or minimal disease activity state are recommended. ${ }^{11}$ In patients with predominantly axial active disease (BASDAI above 4 points) which had insufficient response to NSAIDs, therapy with a bDMARD should be considered. ${ }^{11}$

Assessment of comorbidities is essential in therapy planning and may lead to dosage alteration or restriction of some treatments in the presence of other conditions. ${ }^{10}$ Regarding therapy options in pregnant women, a major principle is to keep a minimal activity state disease while minimising or avoiding drugs that may increase maternal or fetal risk. When considering treatment options in pregnant or breastfeeding women, topical therapies including emollients and low to moderate potency topical steroids are first-line therapy for patients with limited psoriasis. ${ }^{7}$ Systemic medications such as corticosteroids are not regularly used in the treatment of psoriasis, due to common reactivation of symptoms, however they are very effective in cutaneous lesion clearing. ${ }^{7}$ In PsA patients, systemic corticosteroids can be used during pregnancy, especially prednisolone or prednisone. however it is recommended the minimum effective dose concerning best fetal safety profiles. The experience with biological drugs during pregnancy is still limited. ${ }^{7}$

\section{Acknowledgments}

None.

\section{Conflicts of interest}

The author declares there are no conflicts of interest.

\section{Funding details}

None.

\section{References}

1. Singh JA, Guyatt G, Ogdie A et al. Special Article: 2018 American College of Rheumatology/National Psoriasis Foundation Guideline for the Treatment of Psoriatic Arthritis. Arthritis Rheumatol. 2019;71(1):5-32.

2. Brockbank J, Gladman D. Diagnosis and management of psoriatic arthritis. Drugs. 2002;62(17):2447-2457.

3. Taylor W, Gladman D, Helliwell P, et al. CASPAR Study Group. Classification criteria for psoriatic arthritis: development of new criteria from a large international study. Arthritis Rheum. 2006;54(8):2665-2673.

4. Zachariae H. Prevalence of joint disease in patients with psoriasis: implications for therapy. Am J Clin Dermatol. 2003;4(7):441.

5. Boyd AS, Morris LF, Phillips CM, et al. Psoriasis and pregnancy: hormone and immune system interaction. Int J Dermatol. 1996; 35(3):169.

6. Desai RJ, Bateman BT, Huybrechts KF, et al. Risk of serious infections associated with use of immunosuppressive agents in pregnant women with autoimmune inflammatory conditions: cohort study. BMJ. 2017;356:895.

7. Kurizky PS, Ferreira, CC, Nogueira, LSC, et al. Treatment of psoriasis and psoriatic arthritis during pregnancy and breastfeeding. An Bras Dermatol. 2015;90(3).

8. Murase JE, Chan KK, Garite TJ, et al. Hormonal effect on psoriasis in pregnancy and post partum. Arch Dermatol. 2005;141:601.

9. Ursin K, Lydersen S, Skomsvoll JF, et al. Psoriatic Arthritis Disease Activity During and After Pregnancy: A Prospective Multicenter Study. Arthritis Care Res (Hoboken). 2019;71(8):1092-1100.

10. Coates LC, Kavanaugh A, Mease PJ, et al. Group for Research and Assessment of Psoriasis and Psoriatic Arthritis 2015 Treatment Recommendations for Psoriatic Arthritis. Arthritis Rheumatol. 2016;68(5):1060-1071.

11. Gossec L, Smolen JS, Ramiro S, et al. European League Against Rheumatism (EULAR) recommendations for the management of psoriatic arthritis with pharmacological therapies: 2015 update. Ann Rheum Dis. 2016;75(3):499-510. 